\title{
Water-Based Assembly and Purification of Plasmon-Coupled Gold Nanoparticle Dimers and Trimers
}

\author{
Sébastien Bidault ${ }^{1,2}$ and Albert Polman ${ }^{1}$ \\ ${ }^{1}$ Center for Nanophotonics, FOM Institute AMOLF, Science Park 104, 1098 XG Amsterdam, The Netherlands \\ ${ }^{2}$ Institut Langevin, ESPCI ParisTech, CNRS UMR 7587, INSERM U979, 10 Rue Vauquelin, 75005 Paris, France
}

Correspondence should be addressed to Sébastien Bidault, bidault@gmail.com

Received 18 October 2011; Accepted 22 November 2011

Academic Editor: Nicolas Bonod

Copyright ( $\odot 2012$ S. Bidault and A. Polman. This is an open access article distributed under the Creative Commons Attribution License, which permits unrestricted use, distribution, and reproduction in any medium, provided the original work is properly cited.

\begin{abstract}
We describe a simple one-pot water-based scheme to produce gold nanoparticle groupings with short interparticle spacings. This approach combines a cross-linking molecule and a hydrophilic passivation layer to control the level of induced aggregation. Suspensions of dimers and trimers are readily obtained using a single electrophoretic purification step. The final interparticle spacings allow efficient coupling of the particle plasmon modes as verified in extinction spectroscopy.
\end{abstract}

\section{Introduction}

The reproducible synthesis of well-defined metal nanoparticle groupings has been the subject of intense research during the last few years [1-6], driven largely by their use as substrates for surface-enhanced Raman scattering (SERS) [7-10]. The electromagnetic field impinging on pairs of silver or gold particles separated by a few nanometers is enhanced in the gap region by several orders of magnitude [11], allowing for single-molecule sensitivity of the SERS signal $[12,13]$. For a $1 \mathrm{~nm}$ gap, the SERS enhancement factor is larger than $10^{8}$ for gold particles larger than $30 \mathrm{~nm}$ in diameter [7], becoming sufficient to achieve single-molecule SERS sensitivity.

Several approaches have been described using organic linkers $[1-4,6]$ or salt-induced aggregation $[5,9]$ to produce shortly spaced nanoparticle assemblies. In general, particle groupings with controlled geometries and valencies are obtained after several purification and reaction steps. Furthermore, in order to separate dimers and trimers from single particles and larger aggregates, the particles need to be sufficiently stable to sustain electrophoresis [2] or differential centrifugation [5]. Since the colloidal stability of metal nanoparticles decreases for larger diameters, it is difficult to combine efficient purification and large enough particles for SERS.
Electrophoresis was used successfully to purify symmetric or asymmetric gold nanoparticle (AuNP) dimers and trimers linked by DNA strands [2]. Using this technique, we recently demonstrated the controlled assembly of gold nanoparticles smaller than $20 \mathrm{~nm}$ with a $1 \mathrm{~nm}$ gap using a DNA template [7]. Using DNA as a linker offers a large flexibility since a single oligonucleotide can be grafted on the surface of one particle [14]. However, controlled DNA functionalization is difficult to adapt to particles larger than $20 \mathrm{~nm}$ in diameter because of the intrinsic limitations of electrophoresis [15]. In particular, for particles larger than $30 \mathrm{~nm}$ in diameter, the gold surface needs to be passivated before electrophoresis using ethylene glycol oligomers [16]. Furthermore, the DNA single strand grafted on the AuNP must be lengthened during the purification process by hybridizing it to several long strands in order to separate particles conjugated to one biomolecule [17]. Another solution to produce DNA templated groupings with large particles is to increase their size after DNA functionalization by growing a silver shell. This recently allowed single-molecule SERS measurements in a DNA linked particle dimer [10]. In all cases, DNA templated dimers and trimers are obtained after numerous reaction steps carried out over at least 24 hours.

Chen and coworkers recently demonstrated another separation technique of AuNP particle groupings using differential centrifugation [5]. It was used to obtain controlled 
particle nanoassemblies aggregated by salt [5] or organic linkers [6]. However, this approach requires a hydrophobic polymer shell to passivate the particles. Not only does the passivation process require several reaction steps and an organic solvent, the hydrophobic shell also shields the AuNP surface from Raman active molecules. Dye molecules need to be grafted on the gold surface before the passivation and purification steps to obtain a SERS signal [18].

In this paper, we combine fast aggregation and hydrophilic passivation to obtain AuNP dimers and trimers in a water-based one-pot scheme that only requires several minutes. The aggregation process is driven by salt and a dithiol molecule, dithiothreitol (DTT), which provides short interparticle distances. The final hydrophilic ethylene glycol oligomer (PEG) shell allows the electrophoretic purification of particles as large as $35 \mathrm{~nm}$ in diameter. Furthermore, thiolated PEG ligands introduce a negligible background SERS signal on gold particles and are compatible with Raman active molecules $[19,20]$. The combination of large particle diameters and small interparticle spacings yields efficient coupling of the particle plasmon modes as verified in extinction spectroscopy.

\section{Experimental}

2.1. Synthesis of Gold Nanoparticle Dimers. Citrate-coated $35 \mathrm{~nm}$ diameter gold particles were purchased from BBInternational (UK). Bis(p-sulfonatophenyl)phenylphosphine (BSPP) was obtained from Strem Chemicals Europe (France). Thiolated, methyl terminated ethylene glycol oligomers $(n=6)$ were purchased from Polypure (Norway). All other chemicals were obtained from Sigma-Aldrich (USA) and used without further purifications.

A stock solution of AuNPs coated with BSPP is prepared following published procedures $[14,16]$ and is kept for up to 6 months. In brief, $50 \mathrm{~mL}$ of citrate-coated particles are left to incubate overnight with $10 \mathrm{mg}$ BSPP. Particles are then centrifuged to remove the supernatant before being rinsed and finally resuspended in a $0.5 \mathrm{mM}$ BSPP solution. The final AuNP concentration is of the order of $0.02 \mu \mathrm{M}$, and these particles are kept at $8^{\circ} \mathrm{C}$. A typical dimer synthesis is performed using $20 \mathrm{fmol}$ AuNPs in a $50 \mathrm{mM} \mathrm{NaCl}$ solution with a varying dithiothreitol (DTT) concentration (from $1 \mathrm{mM}$ to $40 \mathrm{mM}$ ) and a $10 \mu \mathrm{L}$ reaction volume. The incubation time varies between $1 \mathrm{~s}$ and $10 \mathrm{~min}$ before adding an excess $(100 \mathrm{pmol})$ of thiolated, methylterminated ethylene glycol oligomer (mPEG), which stops the cross-linking effect of DTT by passivating the AuNPs.

The resulting suspensions of single particles and crosslinked groupings are loaded in $1.5 \%$ agarose gels $(0.5 \mathrm{x}$ TrisBorate-EDTA as running buffer) after adding one volume of ficoll loading buffer for four volumes of loaded sample. The gels are run at $8 \mathrm{~V} / \mathrm{cm}$ for 30 mins. The extraction procedure is done following published protocols $[7,14]$, and the sample is concentrated by centrifugation if needed.

2.2. Scanning Electron Microscopy. $2 \mu \mathrm{L}$ of dimer and trimer suspensions obtained after electrophoresis are deposited on freshly cleaned silicon substrates and left to dry before rinsing the sample with $18 \mathrm{M} \Omega$ water and drying it with nitrogen. The samples are imaged using a field emission scanning electron microscope (FEI XL30) operating at $30 \mathrm{keV}$.

2.3. Optical Characterization. Extinction spectra of purified suspensions of dimers and trimers were measured using a dual-beam Genesys $10 \mathrm{~S}$ UV-visible spectrometer (Thermo Fisher Scientific) and compared to a reference $20 \mathrm{pM}$ singleparticle sample.

\section{Results and Discussion}

Dithiothreitol (DTT) was chosen as the cross-linking agent to obtain short interparticle spacings since it features only 5 covalent single bonds (less than $1 \mathrm{~nm}$ long). However, gold nanoparticles coated with a negatively charged phosphine shell (BSPP) and mixed with DTT do not aggregate without charge screening cations. $\mathrm{NaCl}$ is thus required in the reaction buffer to allow a successful interaction. To produce small groupings of particles and not large three-dimensional networks, the assembly process is stopped by adding a thiolated ethylene glycol oligomer (mPEG) that passivates the particle surface and increases the colloidal stability in high salt concentrations. This ligand exchange scheme was used previously to produce DNA functionalized AuNPs with high $\mathrm{NaCl}$ stability $[15,16]$. Our proposed model underlying the fabrication process is summarized in Figure 1. If $\mathrm{NaCl}$ is necessary to drive the particle aggregation process, DTT is necessary to produce stable hydrophilic groupings. Indeed, if the particles are aggregated in salt without DTT, the mPEG surface passivation separates the aggregates back to single particles. Furthermore, if the MPEG excess is left to incubate with the DTT cross-linked particles for several hours, the aggregates are also separated back to single particles. The efficient displacement of the BSPP ligand shell by the mPEG excess can be observed in a Raman spectrum of the AuNPs before and after purification (data not shown): the BSPP SERS response [21] disappears after the ligand exchange process since the mPEG/DTT shell does not produce a measurable Raman signal.

We visualize the formation of particle dimers and trimers by purifying the reaction mix in electrophoresis. In this technique, separation is driven by size and surface charge differences of the different groupings. Electrophoresis also removes unbound ligands and excess salt. We tuned two experimental parameters to optimize the dimer formation: the DTT concentration and the incubation time before adding the mPEG ligand. Figure 2(a) shows the effect of increasing the DTT concentration with a $1 \mathrm{~s}$ incubation time in a $50 \mathrm{mM} \mathrm{NaCl}$ solution. The left lane (lane 1) corresponds to a reference mPEG passivated $35 \mathrm{~nm}$ AuNP sample. Lanes 2 to 4 correspond to DTT concentrations of $1 \mathrm{mM}, 10 \mathrm{mM}$, and $40 \mathrm{mM}$ respectively. New bands are clearly visible in lanes 3 and 4 and correspond to the formation of crosslinked groupings. These bands are also visible using a $1 \mathrm{mM}$ DTT concentration when the incubation time is increased (still with $50 \mathrm{mM} \mathrm{NaCl}$ ) as seen in Figure 2(b) with $1 \mathrm{~s}$, $1 \mathrm{~min}, 2 \mathrm{~min}, 5 \mathrm{~min}$, and $10 \mathrm{~min}$ incubation times. DTTdriven cross-linking is compatible with smaller particles 

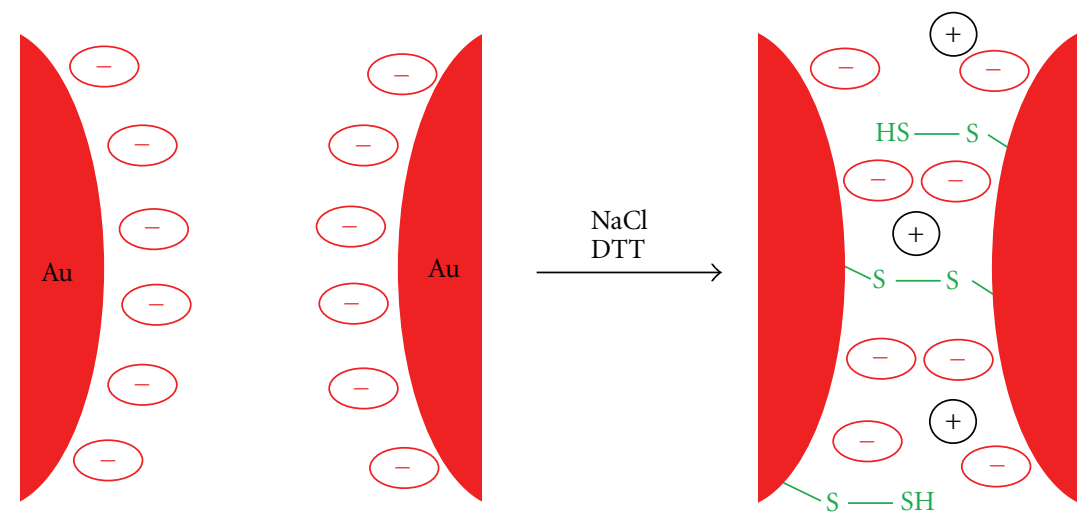

(†)

MPEG

Electrophoresis

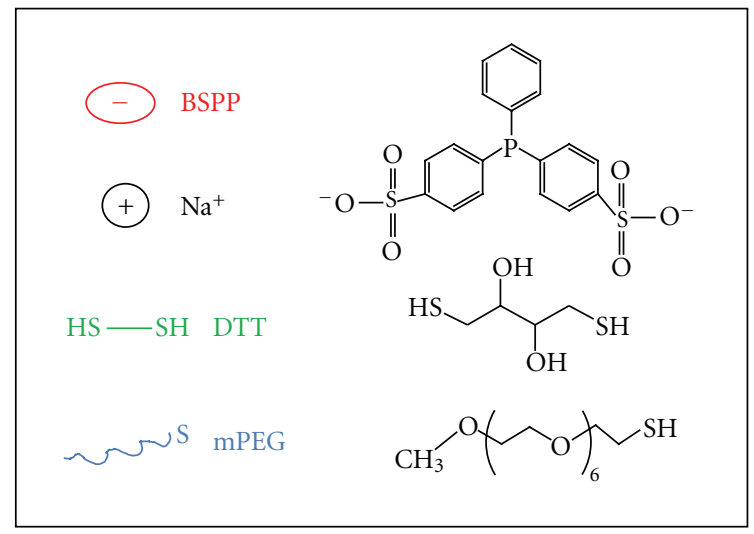

$\downarrow$

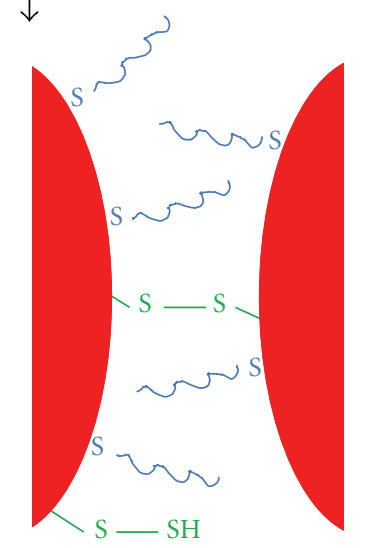

FIGURE 1: Cross-linking scheme. The single linking DTT molecule is only a schematic representation as several molecules can be involved in the dimer formation. The mPEG excess displaces DTT molecules from the gold surface and can thus break interparticle bonds.

by increasing the ionic strength during the aggregation process and adapting the density of agarose gels during electrophoretic purification $(100 \mathrm{mM} \mathrm{NaCl}$ and $2 \%$ agarose gels for $18 \mathrm{~nm}$ diameter AuNPs). Controlled cross-linking of larger gold particles can probably be performed with similar experimental conditions but PEG-stabilized AuNPs larger than $60 \mathrm{~nm}$ do not provide well-defined bands in agarose gel electrophoresis. A different purification process would be necessary.

The first, second, and third fastest bands are recovered from the gel and studied using scanning electron microscopy (SEM). The fastest band, also found on reference samples, only exhibits single particles. The second and third bands correspond preferentially to dimers and trimers as shown in Figures 3(a) and 3(b). The sample purity is estimated by counting the number of particles involved in specific grouping geometries. For the second band, $77 \%$ of the AuNPs are observed in dimers and $23 \%$ as single particles. For the third band, $52 \%$ of the particles form trimers. The rest of the particles are found as dimers $(30 \%)$, single particles $(12 \%)$, or larger aggregates $(6 \%)$. Since the dimer and trimer suspensions are dried on a silicon substrate for the SEM analysis, the interparticle distances observed in electron microscopy can be modified by drying effects. Cryoelectron microscopy would be necessary to observe the particle groupings as they are in suspension [17]. However, the sample purities observed in cryoelectron microscopy and dry SEM are similar, indicating that, at low grouping concentrations, drying effects do not modify significantly the distribution of grouping geometries.

To verify that the short cross-linker allows efficient plasmon coupling necessary for high field enhancements, we measure the extinction spectra of the dimer and trimer suspensions compared to a single-particle sample (all measured in the electrophoresis buffer that does not contain $\mathrm{NaCl}$ ). These extinction spectra are normalized outside of the plasmon resonance frequency $(450 \mathrm{~nm})$ as shown in Figure 4 to correct for the unknown particle concentration in the dimer and trimer samples. The single-particle sample exhibits a typical dipolar plasmon resonance at $525 \mathrm{~nm}$. The dimer and trimer samples exhibit an additional strongly red-shifted side-band that peaks at $630 \mathrm{~nm}$. This band 


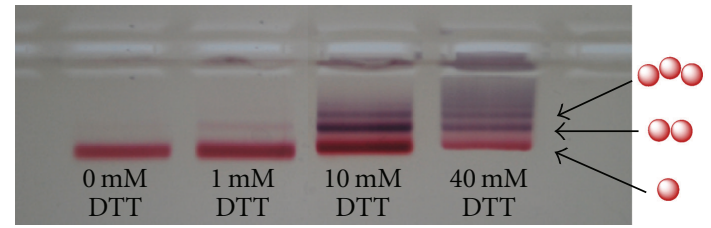

(a)

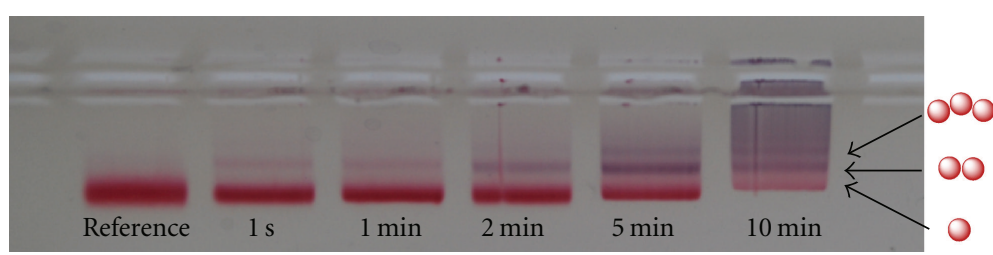

(b)

Figure 2: Agarose gels after electrophoretic purification of reaction mixes. (a) $1 \mathrm{~s}$ incubation time with a $50 \mathrm{mM} \mathrm{NaCl}$ and varying DTT concentrations: $0 \mathrm{mM}$ (lane 1), $1 \mathrm{mM}$ (lane 2), $10 \mathrm{mM}$ (lane 3), and $40 \mathrm{mM}$ (lane 4). (b) $50 \mathrm{mM} \mathrm{NaCl}$ and $1 \mathrm{mM}$ DTT with different incubation times: reference single particles (lane 1), $1 \mathrm{~s}$ (lane 2), $1 \mathrm{~min}$ (lane 3), $2 \mathrm{~min}$ (lane 4), $5 \mathrm{~min}$ (lane 5), and 10 min (lane 6).

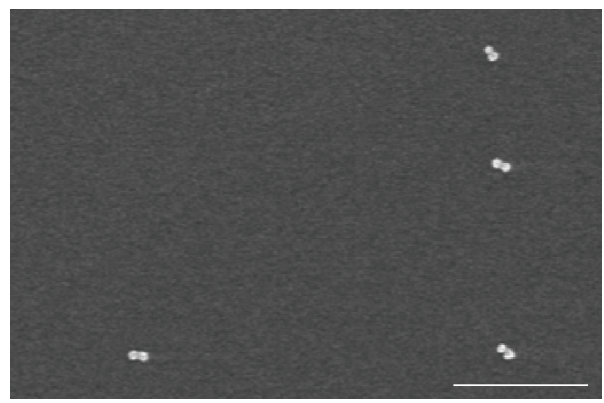

(a)

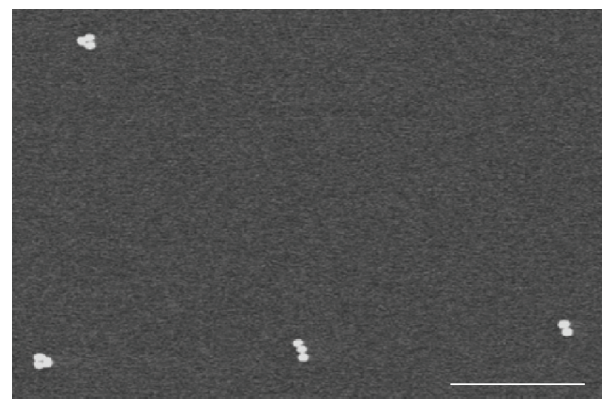

(b)

FIGURE 3: SEM images of dimer (a) and trimer (b) samples deposited on silicon substrates ( $30 \mathrm{keV}$ electrons, scale bar $=500 \mathrm{~nm})$.

corresponds to the longitudinally coupled dipole mode [22, 23]. According to the generalized Mie theory, a $630 \mathrm{~nm}$ longitudinal plasmon resonance for a $35 \mathrm{~nm}$ diameter AuNP dimer in a homogeneous dielectric environment with index 1.4 (intermediate between water and the organic ligand shell [17]) corresponds to an interparticle distance between 1 and $1.5 \mathrm{~nm}$. This distance is consistent with the length of DTT when considering that electrostatic interactions can stretch the groupings in buffers with low ionic strengths [17]. The $630 \mathrm{~nm}$ resonance induces a strong color change in the particle suspension as is clearly visible in Figures 2(a) and 2(b): while the reference single-particle sample is red (lane 1), the slower bands corresponding to the larger AuNP groupings appear purple due to absorption and scattering of both green and red light.

The relative strength of the resonances at $525 \mathrm{~nm}$ and $630 \mathrm{~nm}$ decreases for trimers as they exhibit several longitudinally coupled modes with different phase relations [24]. Furthermore, these resonances are inhomogeneously broadened in the trimer sample since the angle between the particles varies from one grouping to the other (see Figure 3(b)). Similar results were observed in suspensions of $15 \mathrm{~nm}$ diameter AuNP clusters stabilized in block copolymer shells without a cross-linking agent [5]. Overall, these measurements demonstrate that the DTT assembled dimer and trimer suspensions exhibit efficient plasmon coupling and should provide large local field enhancements.

\section{Conclusion}

We have demonstrated that a cross-linking agent, several surface ligand exchange steps, and electrophoresis can be

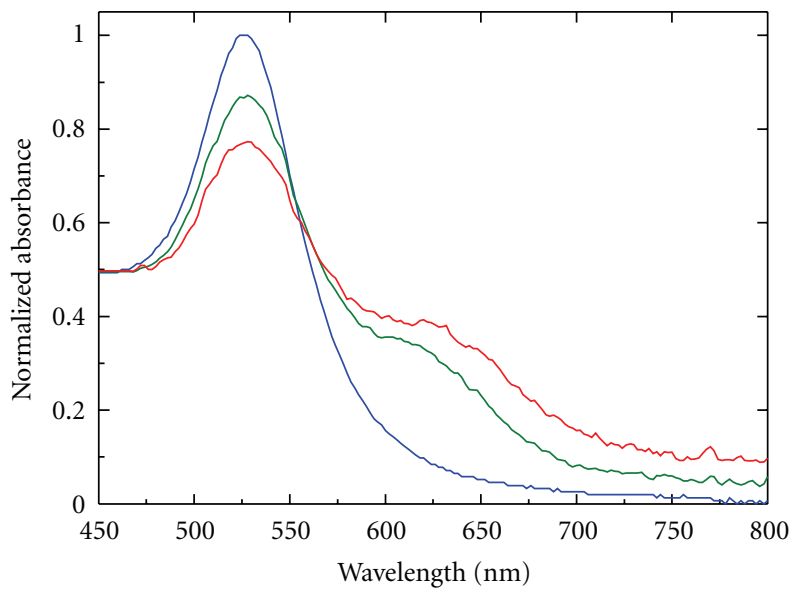

FIGURE 4: Normalized extinction spectra of mPEG stabilized single particles (blue solid line) and electrophoretically purified dimer (green solid line) and trimer (red solid line) suspensions.

used to produce high-purity suspensions of gold particle dimers and trimers with diameters as large as $35 \mathrm{~nm}$ and short interparticle spacings. The overall reaction process only takes a few minutes and the suspensions are obtained with a single purification step. Ensemble extinction measurements demonstrate efficient plasmon coupling in the cross-linked groupings which corresponds to high local field enhancements. By chemically modifying the cross-linking agent to exhibit a molecule with a high Raman cross-section or the binding site of a chosen analyte, it will be possible to produce 
fast and sensitive SERS-based chemical sensors based on this assembly scheme.

\section{Acknowledgments}

Work at AMOLF is part of the research program of FOM which is financially supported by NWO and was also supported by NanoNed, a nanotechnology program of the Dutch Ministry of Economic Affairs.

\section{References}

[1] A. P. Alivisatos, K. P. Johnsson, X. Peng et al., "Organization of 'nanocrystal molecules' using DNA," Nature, vol. 382, no. 6592, pp. 609-611, 1996.

[2] D. Zanchet, C. M. Micheel, W. J. Parak, D. Gerion, S. C. Williams, and A. P. Alivisatos, "Electrophoretic and structural studies of DNA-directed Au nanoparticle groupings," Journal of Physical Chemistry B, vol. 106, no. 45, pp. 11758-11763, 2002.

[3] R. Sardar, T. B. Heap, and J. S. Shumaker-Parry, "Versatile solid phase synthesis of gold nanoparticle dimers using an asymmetric functionalization approach," Journal of the American Chemical Society, vol. 129, no. 17, pp. 5356-5357, 2007.

[4] M. M. Maye, D. Nykypanchuk, M. Cuisinier, D. Van Der Lelie, and O. Gang, "Stepwise surface encoding for high-throughput assembly of nanoclusters," Nature Materials, vol. 8, no. 5, pp. 388-391, 2009.

[5] G. Chen, Y. Wang, L. H. Tan et al., "High-purity separation of gold nanoparticle dimers and trimers," Journal of the American Chemical Society, vol. 131, no. 12, pp. 4218-4219, 2009.

[6] Y. Wang, G. Chen, M. X. Yang et al., "A systems approach towards the stoichiometry-controlled hetero-assembly of nanoparticles," Nature Communications, vol. 1, p. 87, 2010.

[7] S. Bidault, F. J. García De Abajo, and A. Polman, "Plasmonbased nanolenses assembled on a well-defined DNA template," Journal of the American Chemical Society, vol. 130, no. 9, pp. 2750-2751, 2008.

[8] J. Kneipp, X. Li, M. Sherwood et al., "Gold nanolenses generated by laser ablation-efficient enhancing structure for surface enhanced Raman scattering analytics and sensing," Analytical Chemistry, vol. 80, no. 11, pp. 4247-4251, 2008.

[9] W. Li, P. H. C. Camargo, X. Lu, and Y. Xia, "Dimers of silver nanospheres: facile synthesis and their use as hot spots for surface-enhanced raman scattering," Nano Letters, vol. 9, no. 1, pp. 485-490, 2009.

[10] D. K. Lim, K. S. Jeon, H. M. Kim, J. M. Nam, and Y. D. Suh, "Nanogap-engineerable Raman-active nanodumbbells for single-molecule detection," Nature Materials, vol. 9, pp. 60-67, 2010.

[11] H. Xu, J. Aizpurua, M. Käll, and P. Apell, "Electromagnetic contributions to single-molecule sensitivity in surfaceenhanced Raman scattering," Physical Review E, vol. 62, no. 3, pp. 4318-4324, 2000.

[12] K. Kneipp, Y. Wang, H. Kneipp et al., "Single molecule detection using surface-enhanced Raman scattering (SERS)," Physical Review Letters, vol. 78, no. 9, pp. 1667-1670, 1997.

[13] S. Nie and S. R. Emory, "Probing single molecules and single nanoparticles by surface-enhanced Raman scattering," Science, vol. 275, no. 5303, pp. 1102-1106, 1997.
[14] D. Zanchet, C. M. Micheel, W. J. Parak, D. Gerion, and A. P. Alivisatos, "Electrophoretic isolation of discrete au nanocrystal/DNA conjugates," Nano Letters, vol. 1, no. 1, pp. 32-35, 2001.

[15] S. A. Claridge, H. W. Liang, S. R. Basu, J. M. J. Fréchet, and A. P. Alivisatos, "Isolation of discrete nanoparticle-DNA conjugates for plasmonic applications," Nano Letters, vol. 8, no. 4, pp. 1202-1206, 2008.

[16] B. M. Reinhard, S. Sheikholeslami, A. Mastroianni, A. P. Alivisatos, and J. Liphardt, "Use of plasmon coupling to reveal the dynamics of DNA bending and cleavage by single EcoRV restriction enzymes," Proceedings of the National Academy of Sciences of the United States of America, vol. 104, no. 8, pp. 2667-2672, 2007.

[17] M. P. Busson, B. Rolly, B. Stout et al., "Optical and topological characterization of gold nanoparticle dimers linked by a single DNA double-strand," Nano Letters, vol. 11, pp. 5060-5065, 2011.

[18] G. Chen, Y. Wang, M. Yang et al., "Measuring ensembleaveraged surface-enhanced Raman scattering in the hotspots of colloidal nanoparticle dimers and trimers," Journal of the American Chemical Society, vol. 132, no. 11, pp. 3644-3645, 2010.

[19] X. M. Qian, X. H. Peng, D. O. Ansari et al., "In vivo tumor targeting and spectroscopic detection with surface-enhanced Raman nanoparticle tags," Nature Biotechnology, vol. 26, pp. 83-90, 2008.

[20] S. C. Boca and S. Astilean, "Detoxification of gold nanorods by conjugation with thiolated poly(ethylene glycol) and their assessment as SERS-active carriers of Raman tags," Nanotechnology, vol. 21, no. 23, Article ID 235601, 2010.

[21] M. L. Sauthier, R. Lloyd Carroll, C. B. Gorman, and S. Franzen, "Nanoparticle layers assembled through DNA hybridization: characterization and optimization," Langmuir, vol. 18, no. 5, pp. 1825-1830, 2002.

[22] W. Rechberger, A. Hohenau, A. Leitner, J. R. Krenn, B. Lamprecht, and F. R. Aussenegg, "Optical properties of two interacting gold nanoparticles," Optics Communications, vol. 220, no. 1-3, pp. 137-141, 2003.

[23] K. H. Su, Q. H. Wei, X. Zhang, J. J. Mock, D. R. Smith, and S. Schultz, "Interparticle coupling effects on plasmon resonances of nanogold particles," Nano Letters, vol. 3, no. 8, pp. 10871090, 2003.

[24] A. Devilez, B. Stout, and N. Bonod, "Mode-balancing far-field control of light localization in nanoantennas," Physical Review $B$, vol. 81, no. 24, Article ID 245128, 2010. 

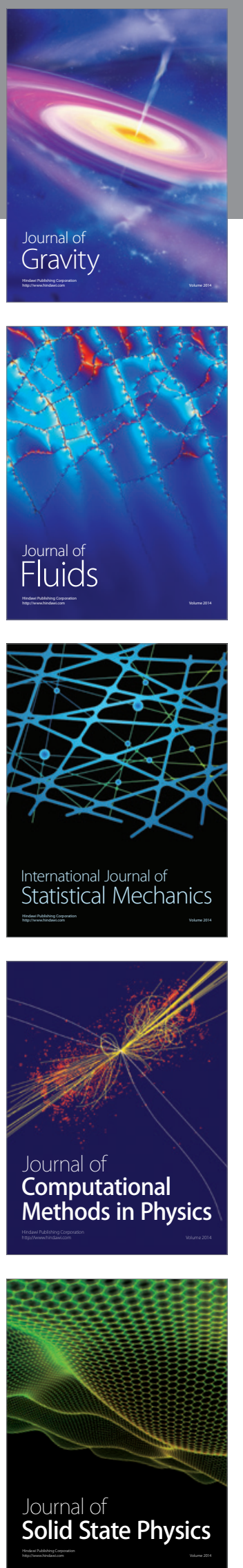

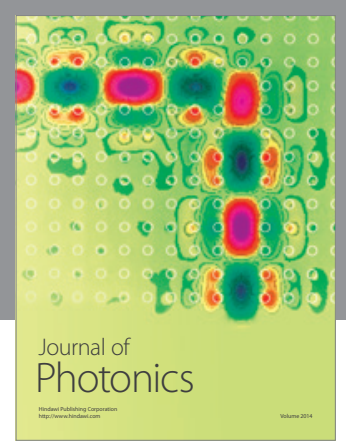

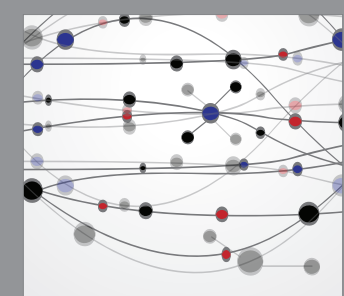

The Scientific World Journal
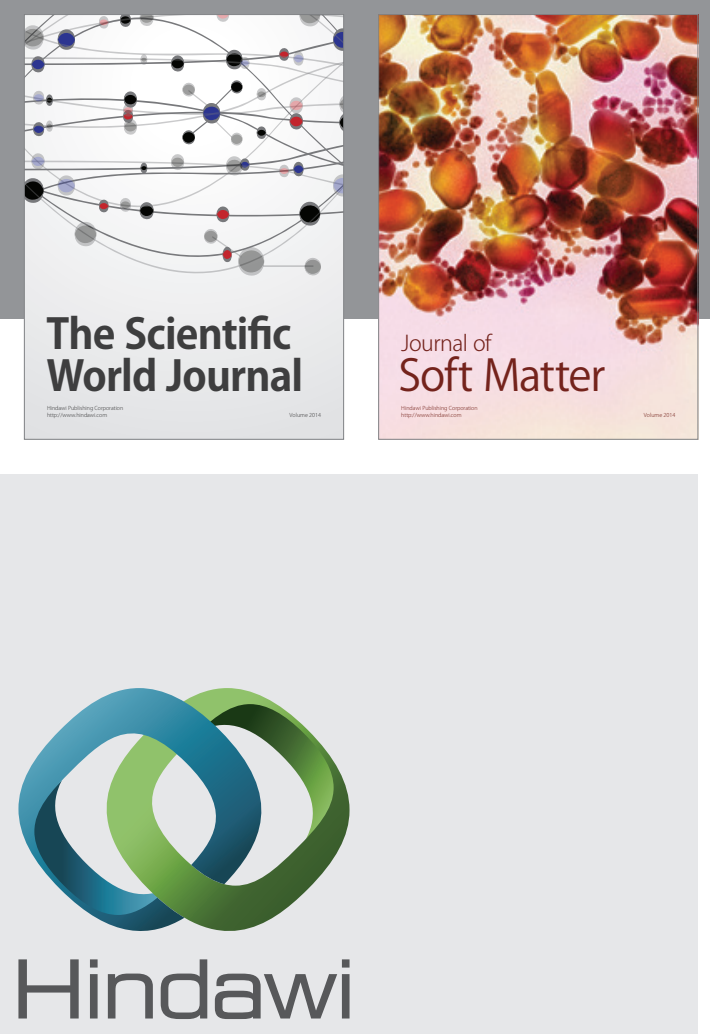

Submit your manuscripts at

http://www.hindawi.com
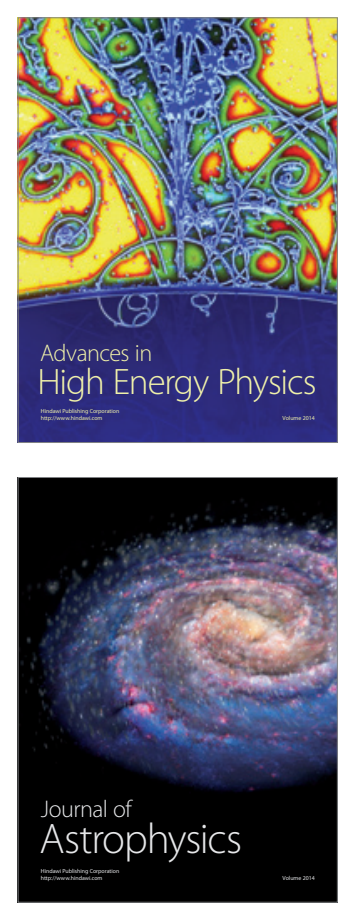
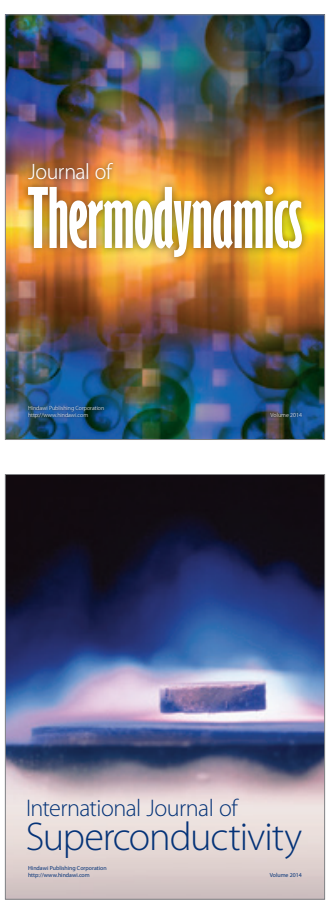
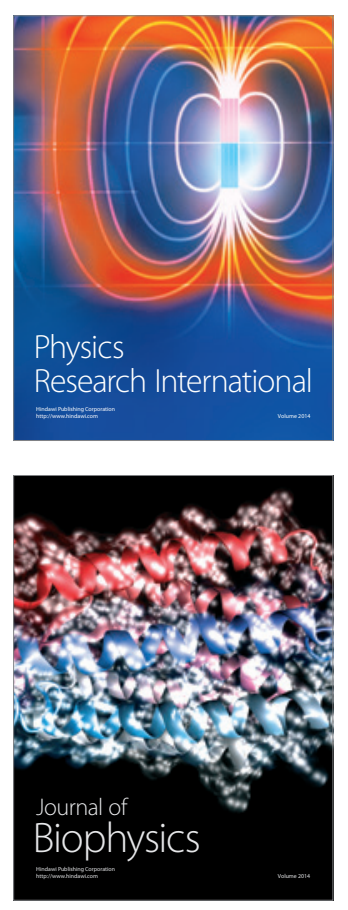
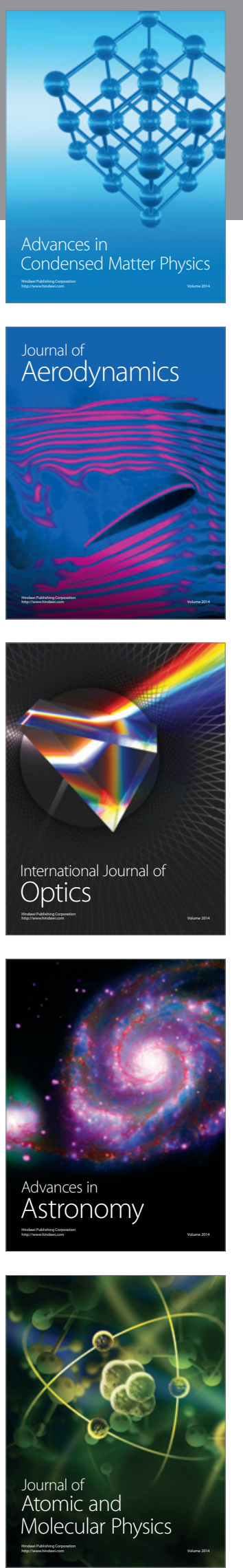